OPEN ACCESS

Edited by: Chun Zhu,

Hohai University, China

Reviewed by: Weibing Gong,

University of California, Berkeley, United States

Xiaqing Qian,

Jiangsu Open University, Japan

*Correspondence:

Shengnian Wang

shengnian.wang@njtech.edu.cn

Specialty section:

This article was submitted to Interdisciplinary Physics,

a section of the journal

Frontiers in Physics

Received: 25 September 2021

Accepted: 19 October 2021

Published: 11 November 2021

Citation:

Cai L, Wang S, Gao X and Li G (2021) Influence of Rock Block Content on

Compaction Characteristics of Graded

Coarse-Grained Fillers.

Front. Phys. 9:782966.

doi: 10.3389/fphy.2021.782966

\section{Influence of Rock Block Content on Compaction Characteristics of Graded Coarse-Grained Fillers}

\author{
Lili Cai ${ }^{1}$, Shengnian Wang ${ }^{2 *}$, Xinqun Gao ${ }^{2}$ and Guoyu $\mathrm{Li}^{3}$ \\ ${ }^{1}$ JSTI Group, Nanjing, China, ${ }^{2}$ College of Transportation Science and Engineering, Nanjing Tech University, Nanjing, China, \\ ${ }^{3}$ State Key Laboratory of Frozen Soil Engineering, Northwest Institute of Eco-Environment and Resources, Chinese Academy \\ of Sciences, Lanzhou, China
}

The bearing capacity, stability, and durability of subgrade are affected by the compaction quality of fillers to a large extent. In this study, samples of graded coarse-grained fillers with different rock block contents were designed and prepared with the aid of an improved continuous gradation equation for coarse-grained soils. Then standard compaction tests of these samples with different water content conditions were conducted to understand the influence of rock block content on the compaction characteristics of graded coarsegrained fillers. Results show that the compaction curve peaks of graded coarse-grained fillers always increase with the rock block content. The maximum dry density of graded coarse-grained fillers increases linearly with the rock block content in approximation, while the optimal water content is the opposite. The optimal water content of graded coarsegrained fillers decreases in nonlinearity with characteristics of rapidly first and then slowly. Two empirical formulas about the rock block content were summarized for predicting the maximum dry density and optimal water content of graded coarse-grained fillers. The results of this study can provide a reference for the engineering application of graded coarse-grained fillers.

Keywords: graded coarse-grained filler, compaction characteristics, rock block content, maximum dry density, optimum water content, empirical formulas

\section{INTRODUCTION}

The compacted coarse-grained fillers have outstanding engineering performances, such as high strength, good stability, and excellent permeability. They have been widely used in earth-rock dams and railway subgrade engineering $[1,2]$. However, engineering performances of such geo-materials are significantly determined by their phase composition and inner structural characteristics. Significant difficulties in parameter determination, especially in compaction quality, always represent a considerable challenge in engineering practices [3].

Scholars from home and abroad have made significant efforts to determine the maximum dry density and optimal water content of coarse-grained fillers, as well as their influence factors $[4,5]$. Some studies by standard compaction tests and surface vibration tests illustrated that the compaction quality of subgrade engineering was significantly affected by their gradation, rock block content, rock block shape, compaction method, and so on. Makedon et al. [6] investigated the influence of gradation on the compaction characteristics of in-situ geo-materials in road construction. Ham et al. [7] studied the influence of gravel content on the compression characteristics of decomposed granite soil. Cavarretta et al. [8] indicated that the behavior of coarse-grained fillers might be slightly 
dependent on the surface roughness and friction, and the influence of particle shape was also very much more significant. Wang et al. [9] indicated that the maximum dry density and optimum water content of crushed sandstonemudstone particle mixtures were increasing then decreasing with the increase of mudstone particle content. Deng et al. [10] found a parabolic relationship between the maximum dry density and coarse grain content and a linear relationship between the optimal water content and coarse grain content for coarse-grained soils. Qiao et al. [4] reported that the size effect, skeleton, and gravel-doped amount were the key factors of compaction characteristics of clay mixed with gravel. Ding et al. [11] pointed out that there was an intrinsic dependence of grain sizes on the maximum dry density for gravelly fillers. Wu et al. [12] studied the effect of gradation on the compatibility of coarse-grained fillers. Several scholars also have discussed the feasibility of empirical prediction methods to indirectly determine the maximum dry density of coarse fillers, such as the elastic wave method and SRM-G prediction model $[13,14]$. All these studies provide a good reference for the engineering performance cognition of coarse-grained fillers. In this study, the graded coarse-grained fillers with different rock block contents were designed and prepared based on an improved continuous empirical gradation equation of coarse-grained fillers, and then standard heavy-duty compaction tests were carried out to explore the influence of rock block content on the compaction characteristics of graded coarse-grained fillers under different water contents. The relationships between the maximum dry density, the optimal water content, and the rock block content of graded coarse-grained fillers were further discussed.

\section{MATERIALS AND METHODS}

\section{Materials and Apparatus}

The coarse-grained fillers can be regarded as a mixture of finegrained fillers and rock blocks. The size threshold $\left(d_{t h r}\right)$ of rock blocks as a function of grain size is always employed to distinguish rock blocks from the fine-grained fillers as

$$
f= \begin{cases}\text { "rockblocks", } & d \geq d_{t h r} \\ \text { "fine fillers", } & d<d_{t h r}\end{cases}
$$

where $d$ is the size of rock blocks or fine-grained filler particles. Some studies have pointed out that the coarse-grained fillers exhibit scale independence in terms of their physical and mechanical properties. Medley and Lindquist [15] summarized an empirical formula based on particle sizes and engineering features as

$$
d_{t h r}=0.05 L_{c}
$$

where $L_{c}$ denotes the characteristic dimension, representing the scale of engineering problems at hand. For three-dimensional problems, the considered diameter of samples is selected as $L_{c}$. In this study, the diameter of samples was $152 \mathrm{~mm}$. So the maximum value of the $d_{t h r}$ should be $7.6 \mathrm{~mm}$. For the sake of conservatism, the value of the $d_{t h r}$ was fixed at $2 \mathrm{~mm}$.
The fine-grained fillers used in this study were clay collected from a highway subgrade in Kunshan, China. The color of this clay was yellowish-brown, and no organic impurities were contained. For the sake of experimental convenience, this clay was dried and sieved to obtain particles lower than $2 \mathrm{~mm}$, and then used to determine its basic physical parameters. The contents of particles ranging 1.0-2.0, 0.63-1.0, 0.63-0.25, $0.25-0.075$ and $<0.075 \mathrm{~mm}$ were $18.24,15.83,23.69,35.03$, and $7.20 \%$. The maximum dry density and optimal water content of this clay were $1.74 \mathrm{~g} / \mathrm{cm}^{3}$ and $16.35 \%$, respectively. Its specified gravity was 2.56 . The natural water content of this clay was $12.44 \%$. The liquid and plastic limits of this clay were 40.23 and $18.58 \%$, respectively. The rock blocks used in the study were crushed gray stone blocks. The density of them was $2.53 \mathrm{~g} /$ $\mathrm{cm}^{3}$. The primary shape of rock blocks was angular. According to the Test Methods of Soils for Highway Engineering (JTG 3430-2020) issued by the Ministry of Transport of the People's Republic of China, a standard heavy-duty compactor II-2 was suggested for compaction tests. The inner diameter and height of the sample tube for this apparatus are 152 and $116 \mathrm{~mm}$, respectively. The height of the protective cylinder is $50 \mathrm{~mm}$. The weight of the compaction hammer was $4.5 \mathrm{~kg}$. The maximum particle size of coarse-grained fillers for this compactor is limited to be $40 \mathrm{~mm}$ for ensuring the accuracy of testing results. So the size of rock blocks was from 2 to $40 \mathrm{~mm}$. Besides, numerous previous experiments and engineering practices indicated that as the compaction work increased, the maximum dry density of soils increased, and the optimal water content decreased [4,5]. Particularly, the maximum dry density of soils would increase nonlinearly with the compaction work. However, when the compaction work reached a certain level, the increase of the maximum dry density of soils almost could be negligible. Therefore, a minimum compaction number on each layer of soil was suggested as 94 in heavy-duty compaction tests. For the reliability of compaction tests on coarse-grained fillers in this study, this number was selected to be 98 .

\section{Testing Scheme and Procedure}

The engineering properties of coarse-grained fillers are affected by rock block content significantly [3]. The rock block content can be determined by the gradation curve with a specified threshold of rock blocks and fine-grained fillers [12,14]. Therefore, the influence of rock block content on compaction characteristics of coarse-grained fillers can come down to their gradations in the final. Some studies pointed out that the properties of coarse-grained fillers will significantly change with the rock block content greater than 20\% [16]. Hence, four kinds of coarse-grained fillers with rock block mass contents of $30,45,60$, and $75 \%$ were considered in this study. Zhu et al. [14] proposed a continuous gradation equation for coarse-grained fillers and proved its broad applicability and superiority in depth. However, the shape parameter determination of that gradation equation depends on massive data fitting. Here, a new improved continuous gradation equation is proposed for being more convenient to apply in practice. 


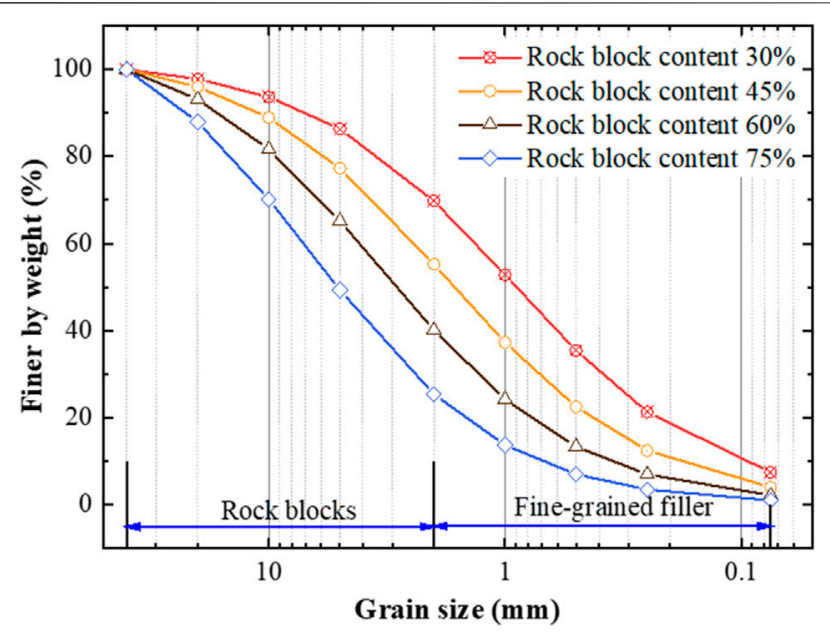

FIGURE 1 | The gradation curves of coarse-grained fillers used in this study.

$$
P=\frac{1}{(1-m)\left(\frac{d_{\max }}{d}\right)^{\frac{1}{\sqrt{m}}}+m}
$$

where $p$ is the proportion of particles smaller than a certain particle size; $d_{\text {max }}$ is the maximum particle size; $m$ is the shape parameter of the gradation curve, $0<m<1$. The greater the value of $m$ is, the more the particles with small size are. The small value of $m$ is the opposite.

According to the above gradation equation and the rock block content design of four kinds of coarse-grained fillers, the gradation curves of coarse-grained fillers used in this study were designed with a maximum rock block size of $40 \mathrm{~mm}$, as shown in Figure 1. The shape parameters of these four gradation curves corresponding to rock block contents of 30,45, 60, and $75 \%$ are $0.978,0.960,0.930$, and 0.875 , respectively. It can be found that the shape parameters of gradation curves are decreasing with the increase of rock block content.

The compaction procedures of coarse-grained fillers are as follows: 1) Smear vaseline on the inner wall of the sample tube and the cushion plate, then connect the sample tube and the protective cylinder. The prepared samples are filled into the sample tube three times and compacted with 98 strikes at each layer. To ensure a good connection, the surface of coarse-grained fillers after compaction was roughened. The final height of the compacted coarse-grained filler sample beyond the top of the sample tube shall not exceed $6 \mathrm{~mm}$ after compaction. 2) Scrape off the excess coarse-grained filler at the top and bottom of the sample tube, and then use an electronic scale to weigh the total mass of the coarsegrained filler sample and the sample tube. 3) Collect testing samples from the center of the compacted coarse-grained filler sample for water content determination. The weighing accurate limits to $0.01 \mathrm{~g}$. The water content of the compacted coarse-grained filler sample should be measured two times. The parallel difference of these testing samples should be no more than 1\%.4) Repeat the above steps for the other tests with pre-arranged water content. 5) Calculate the dry density of compacted coarse-grained filler samples with

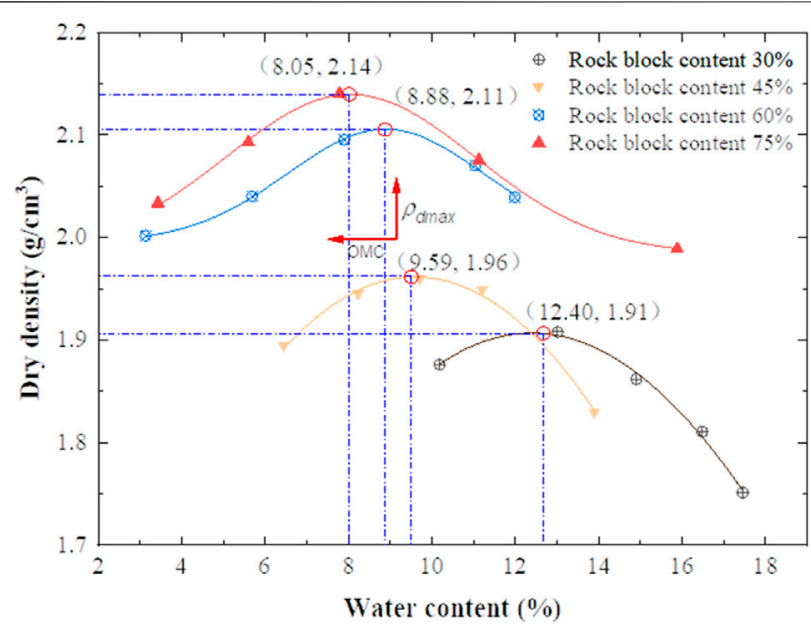

FIGURE 2 | Compaction curves of four designed graded coarse-grained fillers.

different water content, draw compaction curves, and determine the maximum dry density and optimal water content for the tested coarse-grained filler.

\section{Sample Preparation}

During sample preparation, the dosages of rock blocks and finegrained clay were first determined by the designed gradation curves. The sizes of rock blocks were divided into four levels, including 2-5, 5-10, 10-20, and 20-40 mm. Namely, the coarsegrained fillers used in tests were composed of clay and rock blocks with the above-specified sizes. When these materials were prepared, they were dry-mixed fully. The initial water content for sample preparation was pre-selected by the optimal water content of the clay. The water content range and levels were adjusted dynamically according to the compaction test results of the coarse-grained fillers. The water was mixed into the coarsegrained fillers several times in the form of spraying. When the sample preparation is completed, these mixtures were stored in an airtight container for $24 \mathrm{~h}$. The aim of this step, on the one hand, was to distribute the water in coarse-grained fillers evenly, on the other hand, was to ensure the adequate hydration of clay minerals and avoid the influence of the water absorption of rock blocks on the compaction of coarse-grained fillers. Then the prepared coarse-grained fillers were used to conduct compaction tests. All coarse-grained fillers were filled into the compaction sample tube by multiple times and roughened between adjacent layers.

\section{RESULTS AND DISCUSSION Compaction Characteristics of Graded Coarse-Grained Fillers}

Figure 2 shows the compaction results of four designed graded coarse-grained fillers. It can be observed that the dry density of all graded coarse-grained fillers increases first and then decreases 


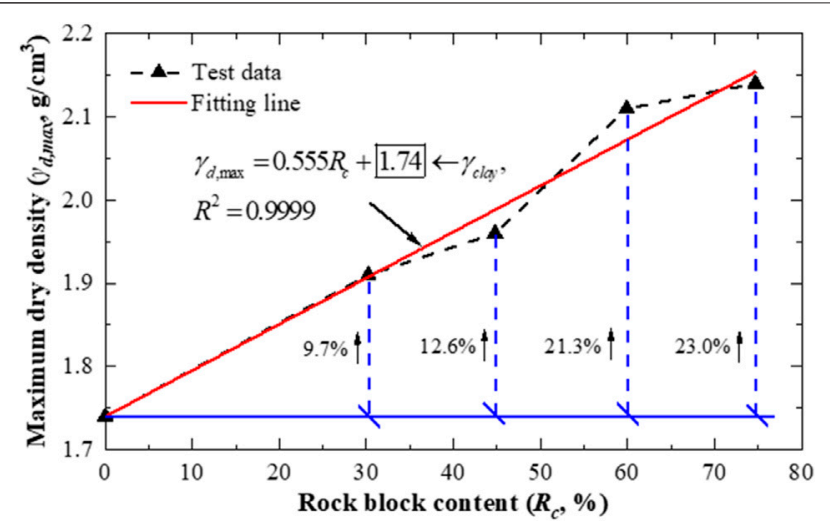

FIGURE 3 | Variation of the maximum dry density of graded coarsegrained fillers with different rock block contents.

with the increase of water content. The graded coarse-grained fillers with $30 \%$ of rock blocks have the highest optimal water content and lowest maximum dry density, while the graded coarse-grained fillers with $75 \%$ of rock blocks are the opposite. The compaction curve peaks of graded coarse-grained fillers rise with the increase of rock block content but shift towards the lower water content. When the rock block content of graded coarsegrained fillers increases from 30 to $75 \%$, their maximum dry densities increases from $1.91 \mathrm{~g} / \mathrm{cm}^{3}$ to $2.14 \mathrm{~g} / \mathrm{cm}^{3}$ with a gradually slowing-down increment. The optimal water content corresponding to the peak of compaction curves decreases from 12.4 to $8.1 \%$ and the reduction amplitude was rather high at the lowest rock block content and then slowed down with the increase of rock block content.

\section{Influence of Rock Block Content on Maximum dry Density}

Figure 3 shows the variation of the maximum dry density of graded coarse-grained fillers with different rock block contents. It can be observed that the maximum dry density of graded coarsegrained fillers increases linearly with the rock block content approximately. Since the density of rock blocks is relatively greater than that of the clay, more rock blocks will contribute to the higher maximum dry density of graded coarse-grained fillers. However, the increasing rate of the maximum dry density of graded coarse-grained fillers does not increase with rock block content in a uniform linear manner. When the rock block content increases from 0 to $30,45,60$, and $75 \%$, the maximum dry density of graded coarse-grained fillers increases by 9.7, 12.6, 21.3, and $23.0 \%$ compared to the case of the pure clay, correspondingly. The reason for this nonuniform increase may be: when the rock block content is lower than $45 \%$, the rock blocks are encased in the clay and do not contact each other directly or completely; the clay is the main contributor to bear the external loading subjected to graded coarse-grained fillers; when the rock block content lies between 45 and $60 \%$, the rock blocks begin to contact and connect to form a relatively stable skeletal structure, replacing the clay as the primary framework of skeleton partially in graded coarse-grained fillers; when the rock block content is greater than $60 \%$, the large amounts of rock blocks with relatively big sizes begin to contact completely; although small rock blocks are filling into skeleton pores among rock blocks and the clay is filled in smaller pores, there are no guarantees for the overhead pores existing in graded coarsegrained fillers. Since the stiffness of rock blocks is relatively higher than that of the fine-grained clay, it is difficult to rearrange the stable skeleton structure of graded coarse-grained fillers at the same compaction loads. The growth rate of the maximum dry density of graded coarse-grained fillers thus decreases. On the whole, the relationship between the maximum dry density $\left(\gamma_{d, \max }\right)$ and rock block content $\left(R_{c}\right)$ of coarse-grained fillers can be described by a linear function as

$$
\gamma_{d, \max }=0.555 R_{c}+1.74 \leftarrow \gamma_{\text {clay }}, \quad R^{2}=0.9999
$$

where the fitting line slope denotes the increasing rate of $\gamma_{d, \max }$, $\gamma_{\text {clay }}$ is the maximum dry density of the clay. The fitting results by Eq. 4 also show that there is a good correlation coefficient for fitting data, which proves the validation of the proposed empirical formula.

\section{Influence of Rock Block Content on Optimal Water Content}

Figure 4 shows the variation of the optimal water content of graded coarse-grained fillers with different rock block contents. It can be observed that the optimal water content of graded coarsegrained fillers has a nonlinear reduction with the increase of rock block content approximately. Namely, the optimal water content of graded coarse-grained fillers changes fast first and then slow with the increase of rock block content. The reason for this nonlinear reduction may be that the increase of rock block content in graded coarse-grained fillers causes the relative content of fine-grained fillers to decrease, thereby resulting in the reduction of free water content and bound water content in the graded coarse-grained fillers. When the rock block content lies between 0 and $45 \%$, namely the clay content in graded coarsegrained fillers is more than rock blocks, the water in graded coarse-grained fillers is more hidden in the clay by the form of

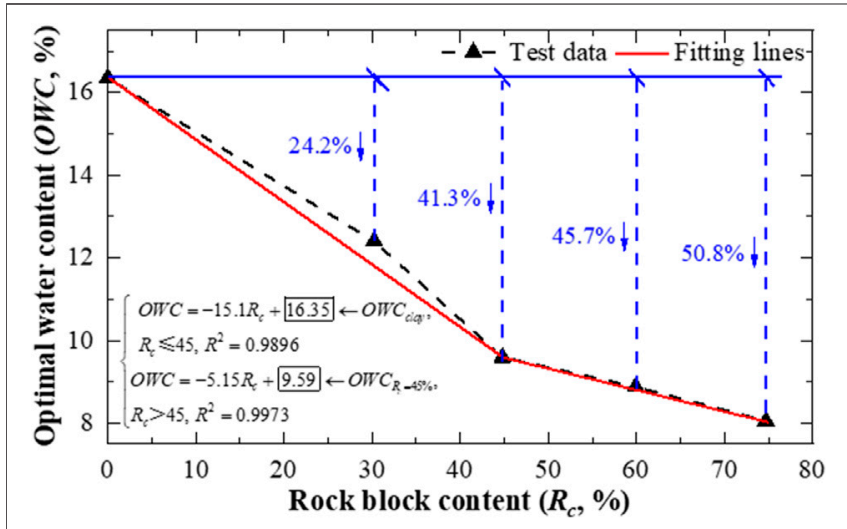

FIGURE 4 | Variation of the optimal water content of graded coarsegrained fillers with different rock block contents. 
combing free water and bound water. The relative content of the clay thus primarily controls the optimal water content of graded coarse-grained fillers. However, the relative content of the clay in graded coarse-grained fillers will decrease rapidly with the increase of rock block content and the water absorption of rock blocks is always limited. So the optimal water content of graded coarse-grained fillers decreases with the increase of rock block content following a gradually reduced amplitude. However, the relationship between the optimal water content $(O W C)$ and rock block content $\left(R_{c}\right)$ of coarse-grained fillers can also be approximately described by piecewise linear functions as

$$
\left\{\begin{array}{l}
O W C=-15.1 R c+16.35 \leftarrow O W C_{\text {clay }}, \quad R c \leq 45, \quad R^{2}=0.9896 \\
O W C=-5.15 R c+9.59 \leftarrow O W C_{R_{c}=45 \%,} \quad R c>45, \quad R^{2}=0.9973
\end{array}\right.
$$

where $O W C_{\text {clay }}$ denotes the optimal water content of the clay, $O W C_{R c=45 \%}$ denotes the optimal water content of coarse-grained fillers with the rock block content of $45 \%$. The high fitting correlation coefficients of these two piecewise linear functions show their feasibility for predicting the optimal water content of coarse-grained fillers.

\section{CONCLUSION}

The compacted coarse-grained fillers are ideal subgrade fillers with outstanding engineering performances. However, their complex composition and inner structure characteristics cause great difficulties in parameter determination. In this study, the compaction characteristics of graded coarse-grained fillers with different rock block contents were investigated by a standard heavy-duty compactor first and then the influence of rock block content on the maximum dry density and optimal water content of graded coarse-grained fillers was discussed. The results show that the maximum dry density of graded coarse-grained fillers increases linearly with the rock bock content approximately but

\section{REFERENCES}

1. Fei K. Experimental Study of the Mechanical Behavior of clay-aggregate Mixtures. Eng Geology (2016) 210:1-9. doi:10.1016/j.enggeo.2016.05.011

2. Chen RP, Qi S, Wang HL, Cui YJ. Microstructure and Hydraulic Properties of Coarse-Grained Subgrade Soil Used in High-Speed Railway at Various Compaction Degrees. J Mater civil Eng (2019) 31(12):4019301. doi:10.1061/ (ASCE)MT.1943-5533.0002972

3. Wang S, Li Y, Gao X, Xue Q, Zhang P, Wu Z. Influence of Volumetric Block Proportion on Mechanical Properties of Virtual Soil-Rock Mixtures. Eng Geology (2020) 278(1):105850. doi:10.1016/j.enggeo.2020.105850

4. Qiao L, Pang L, Li Y, Sui Z. Experimental Study of Compaction Characteristics of Oversized clay Mixed with Gravels. Chin J Rock Mech Eng (2014) 33(3): 484-92. doi:10.13722/j.cnki.jrme.2014.03.005

5. Sharma B, Gogoi B, Sridharan A. Static Compaction Characteristics of Coarse and Fine Grained Soils. Cham: Springer International Publishing (2018). p. 45-57. doi:10.1007/978-3-319-95792-0_4

6. Makedon T, Tremopoulou E, Dimopoulos G. The Influence of Gradation on the In Situ Compaction of Geo-Materials in Road Construction. Bull Eng Geol Environ (2009) 68(1):81-7. doi:10.1007/s10064-008-0176-8 posses a nonuniform linear increased amplitude. The graded coarse-grained fillers with the rock block content ranging from 45 to $60 \%$ have the most significant change due to the forming relatively stable skeletal structure. The optimal water content of graded coarse-grained fillers decreases nonlinearly with the increase of rock block content and their reduced amplitudes are fast first and then slow with the increase of relative content of rock blocks due to limited water absorption of rock blocks. Two empirical formulas about the rock block content were summarized for predicting the maximum dry density and optimal water content of graded coarse-grained fillers. The results of this study can provide a reference for the application of graded coarse-grained fillers in subgrade engineering.

\section{DATA AVAILABILITY STATEMENT}

The original contributions presented in the study are included in the article/Supplementary Material, further inquiries can be directed to the corresponding author.

\section{AUTHOR CONTRIBUTIONS}

Experimental design and review of the work, LC; Funding acquisition and formal writing of the work, SW; investigation and data analysis of the work, XG; Review of the work, GL. All authors have read and agreed to the published version of the manuscript.

\section{FUNDING}

This work was supported by the National Natural Science Foundation of China (41902282); State Key Laboratory of Frozen Soil Engineering (SKLFSE201809).

7. Ham T-G, Nakata Y, Orense RP, Hyodo M. Influence of Gravel on the Compression Characteristics of Decomposed Granite Soil. J Geotech Geoenviron Eng (2010) 136(11):1574-7. doi:10.1061/(asce)gt.19435606.0000370

8. Cavarretta I, Coop M, O'Sullivan C. The Influence of Particle Characteristics on the Behaviour of Coarse Grained Soils. Géotechnique (2010) 60(6):413-23. doi:10.1680/geot.2010.60.6.413

9. Wang J-J, Zhang H-P, Deng D-P, Liu M-W. Effects of Mudstone Particle Content on Compaction Behavior and Particle Crushing of a Crushed sandstone-mudstone Particle Mixture. Eng Geology (2013) 167:1-5. doi:10.1016/j.enggeo.2013.10.004

10. Deng G, Zhang J, Meng F, Wang S, Shi X. Static Characteristics of CoarseGrained Soils. Electron J Geotechnical Eng (2014) 19:1977-90. Available from: http://www.ejge.com/2013/Ppr2013.464nar.pdf.

11. Ding Y, Rao Y, Sarmah AK, Huang X, Pan B, Liu D. Prediction and Evaluation of Grain Size-dependent Maximum Dry Density for Gravelly Soil. Int J Geomechanics (2020) 20(9):4020153. doi:10.1061/(ASCE)GM.19435622.0001775

12. Wu E, Zhu J, Guo W, Zhang Z. Effect of Gradation on the Compactability of Coarse-Grained Soils. KSCE J Civ Eng (2020) 24(2):356-64. doi:10.1007/ s12205-020-1936-7 
13. Zhang XM, Yao-Zhi L, Dong Q, Zhang YH. Evaluating Compaction Quality of Soil-Rock Mixture Based on Theory of Elastic Waves. Chin J Geotechnical Eng (2015) 37:2051. doi:10.11779/CJGE201511016

14. Zhu JG, Guo WL, Wen YF, Yin JH, Zhou C. New Gradation Equation and Applicability for Particle-Size Distributions of Various Soils. Int J Geomechanics (2018) 18(2):4017155. doi:10.1061/(ASCE)GM.19435622.0001082

15. Medley E, Lindquist ES. The Engineering Significance of the Scaleindependence of Some Franciscan Melanges in California, USA. In: The 35th U.S. Symposium on Rock Mechanics. Reno, Nevada: USRMS (1995).

16. Wang SN, Shi C, Xu WY, Wang HL, Zhu QZ. Numerical Direct Shear Tests for Outwash Deposits with Random Structure and Composition. Granular matter (2014) 16(5):771-83. doi:10.1007/s10035-014-0504-6

Conflict of Interest: Author LC was employed by the company JSTI Group.
The remaining authors declare that the research was conducted in the absence of any commercial or financial relationships that could be construed as a potential conflict of interest.

Publisher's Note: All claims expressed in this article are solely those of the authors and do not necessarily represent those of their affiliated organizations, or those of the publisher, the editors and the reviewers. Any product that may be evaluated in this article, or claim that may be made by its manufacturer, is not guaranteed or endorsed by the publisher.

Copyright $\odot 2021$ Cai, Wang, Gao and Li. This is an open-access article distributed under the terms of the Creative Commons Attribution License (CC BY). The use, distribution or reproduction in other forums is permitted, provided the original author(s) and the copyright owner(s) are credited and that the original publication in this journal is cited, in accordance with accepted academic practice. No use, distribution or reproduction is permitted which does not comply with these terms. 